\title{
Sponsoring: Unklarheit trotz Transparenzgesetz
}

\author{
Zuwendungen der Pharmaindustrie an die Autoren von Leitlinien müssen in den USA von \\ beiden Seiten offengelegt werden. Dabei tritt eine bemerkenswerte Diskrepanz auf.
}

_ Autoren medizinischer Leitlinien müssen in den USA alle Zuwendungen der Industrie als mögliche Interessenkonflikte angeben. Seit 2011 sind auch alle Pharma- und Gerätehersteller verpflichtet, Zahlungen an Ärzte und Kliniken anzuzeigen. Dies wird im Internet veröffentlicht. Jetzt hat man die Angaben von 588 Autoren, die an 39 Leitlinien der beiden US-Fachgesellschaften für Kardiologie mitwirkten, mit jenen von 15 Sponsoren verglichen.

Die generelle Übereinstimmung war schlecht. Insgesamt versäumten 55\% der Autoren die Angabe einer Zuwendung, während $72 \%$ der Zuwendungen von der Industrie nicht deklariert wurden. Auch in den einzelnen Kategorien Vortragshonorare, Beratung und Forschung waren die Angaben der Industrie unzuverlässiger als die der Autoren. Nur bei sonstigen Zuwendungen wie Reisekosten, Mahlzeiten und Lizenzgebühren waren die Autoren ungenauer.

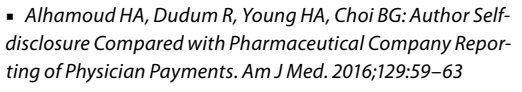

- Alhamoud HA, Dudum R, Young HA, Choi BG: Author Selfdisclosure Compared with Pharmaceutical Company Reporting of Physician Payments. Am J Med. 2016;129:59-63

\section{KOMMENTAR}

Im Jahr 2012 gingen 4 Milliarden US-Dollar von der Industrie an die Kardiologie. Weil befürchtet wird, dass solche Zahlungen auch zu Interessenkonflikten führen und das Verschreibungsverhalten beeinflussen können, wurde mit dem "Sunshine Act" die gesetzliche Berichtspflicht der Sponsoren eingeführt. Schon länger bestehen Fachzeitschriften und - gesellschaften darauf, dass Autoren Zuwendungen der Industrie als mögliche Interessenkonflikte offenlegen. Viele Gesellschaften haben Regeln für Leitlinienkommissionen z. B. muss die Hälfte der Autoren „zuwen- dungsfrei" sein, und der Vorsitzende darf zumindest in den letzten zwei Jahren keine erhalten haben.

Der "Sunshine Act" sollte Transparenz schaffen. Die problemlose Überprüfbarkeit sollte verhindern, dass Interessenkonflikte verschwiegen werden. Die erheblichen Diskrepanzen zwischen den Angaben von Ärzten und Industrie sind deshalb bemerkenswert. Die Autoren führen dies weniger auf vorsätzliche Falschaussagen, sondern auf Regelungsmängel und Ermessenfragen zurück. Wann „verjährt" eine Zuwendung? Ab welchem Wert wird eine Zuwendung deklarationspflichtig? Eine Diskrepanz ist auch programmiert, wenn ein Sponsor die Teilnehmer einer Fortbildungsveranstaltung zum Abendessen einlädt und das entsprechend deklariert, doch viele Ärzte nach einem anstrengenden Tag lieber nach Hause eilen.

Prof. Dr. med. H. Holzgreve

\section{Bei diesem Patienten nagt die Aorta am Knochen}

Ein 74-jähriger Mann stellte sich wegen akuter Rückenschmerzen vor. Sein Blutdruck war trotz Hypertonie-Anamnese aktuell normal. Jahre zuvor war er wegen eines infrarenalen Bauchaortenaneurysmas operiert worden. Bei terminaler Niereninsuffizienz war er dialysepflichtig. Im CT von Thorax und Abdomen zeigte sich ein großes thorakales Aortenaneurysma in der Ausdehnung von $8,1 \times 11,7 \mathrm{~cm}$ in der axialen Schnittführung, das zu Erosionen der Brustwirbelkörper 10 und 11 geführt hatte (Abb. A-C).
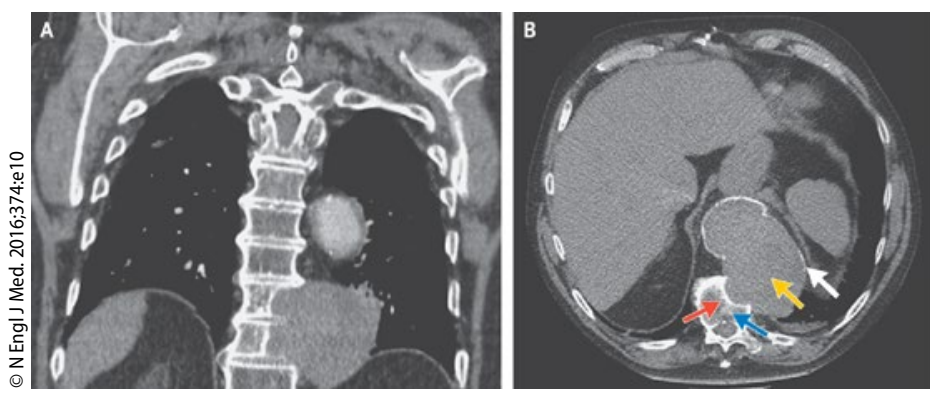

Erosionen (rote Pfeile) der BWK 10 und 11 (blauer Pfeil) durch ein thorakales Aortenaneurysma (gelbe Pfeile); erkennbar sind feine, ringförmige Verkalkungen (weiße Pfeile).

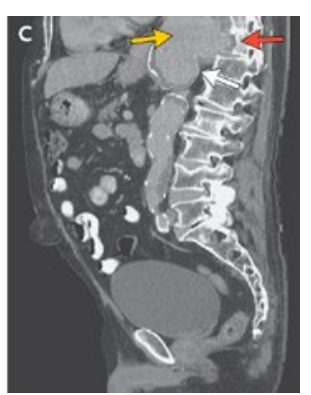

Erosionen von Brustwirbelkörpern sind eine seltene Komplikation eines thorakalen Aortenaneurysmas. Häufiger sieht man sie bei einem Ersatz des Aortenbogens. Als pathogenetischen Mechanismus nimmt man eine Ernährungsstörung des Knochens bei wiederholter mechanischer Kompression und relativer Ischämie an. Differenzialdiagnostisch kommen retroperitoneale Raumforderungen bei Tumoren oder Infektionen in Betracht. Die erhaltenen Zwischenwirbelräume, wie sie bei diesem Patienten zu sehen sind, sprechen dagegen, dass es sich um eine Infektion handelt. Ebenfalls typisch für das thorakale Aortenaneurysma ist die feine, ringförmige Verkalkung (Abb. B, C).

Wegen des schlechten Allgemeinzustands wurde der Patient nicht operiert. Unter konservativer Therapie lebte er noch drei Jahre. Er starb nach einem kurzen Schmerzrezidiv.

Prof. Dr. med. H. S. FüeßI

-Wansink J, van der Kleij FGH (j.wansink@gmail.com). Vertebral-body erosion in thoracic aortic aneurysm. NEngl J Med. 2016;374:e10 